\title{
Commentary: Pharmacological Interventions for Bacterial Prostatitis
}

\author{
Vittorio Magri ${ }^{1}$, Konstantinos Stamatiou ${ }^{2}$, Alberto Trinchieri ${ }^{3}$ and Gianpaolo Perletti ${ }^{4,5 *}$ \\ 1 Urology Clinic, ASST Nord, Milano, Italy, ${ }^{2}$ Urology Department, Tzaneio General Hospital, Piraeus, Greece, ${ }^{3}$ Urology Unit, \\ Manzoni Hospital, Lecco, Italy, ${ }^{4}$ Section of Medical and Surgical Sciences, Department of Biotechnology and Life Sciences, \\ University of Insubria, Varese, Italy, ${ }^{5}$ Department of Human Structure and Repair, Ghent University, Ghent, Belgium
}

Keywords: prostatitis, chronic bacterial prostatitis, fluoroquinolones, macrolides, combination therapy

\section{A Commentary on}

Pharmacological Interventions for Bacterial Prostatitis

By Xiong S, Liu X, Deng W, Zhou Z, Li Y, Tu Y, Chen L, Wang G and Fu B. Front Pharmacol (2020). 11:504. doi: 10.3389/fphar.2020.00504.

\section{OPEN ACCESS}

Edited by:

Heike Wulf,

University of California, Davis, United States

Reviewed by: Kurt G. Naber, Technical University of Munich,

Germany

Sandor Kerpel-Fronius, Semmelweis University, Hungary

${ }^{*}$ Correspondence:

Gianpaolo Perlett gianpaolo.perletti@uninsubria.it

Specialty section: This article was submitted to Pharmaceutical Medicine and

Outcomes Research

a section of the journal

Frontiers in Pharmacology

Received: 18 June 2020 Accepted: 15 September 2020 Published: 06 October 2020

Citation:

Magri V, Stamatiou K, Trinchieri A and Perletti G (2020) Commentary: Pharmacological Interventions for Bacterial Prostatitis.

Front. Pharmacol. 11:573903. doi: 10.3389/fphar.2020.573903
In a recent issue of this journal, Xiong and coworkers published a comprehensive article focusing on pharmacological interventions for bacterial prostatitis (Xiong et al., 2020).

After reviewing all available evidence about the etiology and current therapeutic options for both acute and chronic presentations of bacterial prostatitis, the authors provided useful diagnostic and therapeutic algorithms for both conditions.

In their algorithm, Xiong et al. include combination therapy (and in particular antibacterials combined with other antibacterials) as a possible option in case of unsatisfactory results of singleagent treatment of chronic bacterial prostatitis (CBP). According to our experience, combination of fluoroquinolones with other antibacterial agents is indeed a viable and effective therapeutic strategy, which may also hinder the emergence of resistant pathogens, in a time of dramatic increase of resistance to first-choice fluoroquinolone antibacterials (FQs).

As a caveat to the algorithm for CBP included in the excellent review by Xiong and coworkers, we deem important to emphasize that physicians who intend to adopt such therapeutic approach must be aware of the potential risks associated with the combination of a fluoroquinolone with a macrolide antibiotic. Since certain FQs and macrolides can cause the elongation of the QT interval of the electrocardiogram, candidate patients should be carefully interviewed and their cardiological profile evaluated before starting a course of therapy, as indicated in detail in a previous article (Magri et al., 2019). Notably, a history of long QT syndrome, or ongoing therapy with agents known to elongate the QT interval (for a list of such agents, see for instance: Tisdale, 2016) may be exclusion criteria, as addition of a FQ and/or a macrolide may increase in certain categories of patients (the elderly, for example) the risk of heart rhythm disorders (Choi et al., 2018; Gorelik et al., 2019).

Warnings considerably restricting the usage of FQs due to the possible emergence of severe adverse effects (e.g., an increased risk of neuropathy, depression, memory impairment, other neurological disorders, aneurysm, tendon rupture) have been recently published by the FDA and by the European Medicine Agency (European Medicine Agency, 2018; Food and Drug Administration, 2018). However, whereas empirical administration of FQs for abacterial prostatitis is no longer authorized (European Medicine Agency, 2018), FQs like ciprofloxacin or levofloxacin continue to be approved for use in patients with documented bacterial prostatitis in the USA, in the European Union and in other 
countries (e.g., the United Kingdom). This decision is probably due to the fact that FQs are the best available therapy for CBP in terms of antibacterial spectrum, prostatic distribution and potency, and that very few agents (trimethoprim, macrolides, carbapenems) are distributed to prostatic ducts and interstitial spaces, achieving concentrations above the MIC of Gram-negative and Grampositive pathogens. However, since it is known that (i) pathogen eradication rates by trimethoprim are not optimal (about $60 \%$ ), that (ii) macrolides do not have a broad spectrum of antibacterial activity, and (iii) that the use of carbapenems (the only betalactams which are sufficiently distributed to the prostate) is now restricted to the hospital setting, fluoroquinolones necessarily remain a mainstay of CBP therapy in the outpatient setting.

Given the restrictions listed above, and in the presence of worrisome fluoroquinolone resistance scenarios, research groups worldwide are investigating the efficacy of alternative antibiotics. In their review, Xiong and coworkers have summarized evidence suggesting that fosfomycin may become in the next future a safe and effective agent for treatment of CBP, especially in the presence of multidrug-resistant Enterobacteriaceae. Whereas fosfomycin is also available as an intravenous preparation, the vast majority of the published studies refer to the oral intake of the drug (3 grams daily, single-dose). Consensus about the optimal dosing of this agent has not been reached yet. According to our experience, a "switch protocol" similar to the one implied by Xiong et al. and adopted by Karaiskos et al. (oral fosfomycin $3 \mathrm{~g} /$ day for 7-10 days, switched to $3 \mathrm{~g}$ q48h for 6 weeks) can address at the same time the need for a "full-dosage hit" at the start of treatment, and the necessity to prevent/control diarrhea (the most common side effect of this drug) for the subsequent weeks of therapy (Karaiskos et al., 2019; Stamatiou et al., 2019). Adequately powered randomized studies are warranted to provide quality evidence concerning the effectiveness and optimal dosing of this antibiotic. Combination of fosfomycin with other antibacterial agents, possibly hindering the emergence of chemoresistance in

\section{REFERENCES}

Choi, Y., Lim, H. S., Chung, D., Choi, J. G., and Yoon, D. (2018). Risk evaluation of azithromycin-induced QT prolongation in real-world practice. BioMed. Res. Int. 2018, 1574806. doi: 10.1155/2018/1574806

European Medicine Agency (2018). Disabling and potentially permanent side effects lead to suspension or restrictions of quinolone and fluoroquinolone antibiotics. Available at: https://www.ema.europa.eu/en/documents/pressrelease/disabling-potentially-permanent-side-effects-lead-suspensionrestrictions-quinolone-fluoroquinolone_en.pdfhttps://www.fda.gov/media/ 119532/download (Accessed June 12, 2020).

Food and Drug Administration (2018). Safety announcement. Available at: https:// www.fda.gov/media/119532/download (Accessed June 12, 2020).

Gorelik, E., Masarwa, R., Perlman, A., Rotshild, V., Abbasi, M., Muszkat, M., et al. (2019). Fluoroquinolones and cardiovascular risk: a systematic review, Metaanalysis and network meta-analysis. Drug Saf. 42, 529-538. doi: 10.1007/ s40264-018-0751-2

Karaiskos, I., Galani, L., Sakka, V., Gkoufa, A., Sopilidis, O., Chalikopoulos, D., et al. (2019). Oral fosfomycin for the treatment of chronic bacterial prostatitis. J. Antimicrob. Chemother. 74, 1430-1437. doi: 10.1093/jac/ dkz015

Magri, V., Perletti, G., Cai, T., Stamatiou, K., Trinchieri, A., and Montanari, E. (2019). Levofloxacin for NIH Category II Chronic Bacterial Prostatitis: A RealLife Study. Chemotherapy 64, 8-16. doi: 10.1159/000499034 pathogens, is highly desirable. For the moment, published evidence in this respect is missing. According to our preliminary experience, pathogen eradication and symptom remission has been achieved by combining fosfomycin with other agents, although we cannot attribute therapeutic success to the drug combination or to the prominent action of a single agent. In a series of 8 patients treated with fosfomycin and levofloxacin for 4 weeks, six patients (E. coli, n=4; E. faecalis, $\mathrm{n}=2$ ) showed pathogen eradication and symptom resolution, whereas 2 mixed infections by Enterococcus and E. coli showed pathogen and symptom persistence. When fosfomycin was combined with beta-lactams characterized by limited prostatic penetration (co-amoxiclav, $n=6$; ampicillin-sulbactam, $n=2$ ), in the attempt of eradicating Grampositive infections (E. faecalis, with or without concomitant E. coli and Staphylococci), pathogen eradication and symptom remission was achieved in all patients. As case series like this are only minimally indicative of some degree of efficacy, adequately powered studies in a randomized setting must have a final say on this issue.

According to the World Health Organization (WHO), lack of innovation in the development of new antibiotics are undermining efforts to combat drug-resistant infections, and the pipeline for antibacterial agents targeting Gram-negative pathogens appears to be particularly weak (World Health Organization, 2019). In the face of a worrisome decline of private investment in this field, public academic and non-academic research institutions represent our last hope in this regard.

\section{AUTHOR CONTRIBUTIONS}

GP and AT searched the literature and conceived and wrote the article. VM and KS critically appraised the literature and made an intellectual contribution to the work. All authors contributed to the article and approved the submitted version.

Stamatiou, K., Perletti, G., Magri, V., and Trinchieri, A. (2019). Usage and dosage of fosfomycin for NIH category II chronic bacterial prostatitis. Hellenic Urol. 31, 42-49. doi: 10.1093/jac/dkz015

Tisdale, J. E. (2016). Drug-induced QT interval prolongation and torsades de pointes: role of the pharmacist in risk assessment, prevention and management. Can. Pharm. J. 149, 139-152. doi: 10.1177/1715163516641136

World Health Organization (2019). Antibacterial agents in clinical development: an analysis of the antibacterial clinical development pipeline. (Geneva: World Health Organization). Available at: https://apps.who.int/iris/bitstream/handle/ 10665/330420/9789240000193-eng.pdf.

Xiong, S., Liu, X., Deng, W., Zhou, Z., Li, Y., Tu, Y., et al. (2020). Pharmacological interventions for bacterial prostatitis. Front. Pharmacol. 11, 504. doi: 10.3389/ fphar.2020.00504

Conflict of Interest: The authors declare that the research was conducted in the absence of any commercial or financial relationships that could be construed as a potential conflict of interest.

Copyright (c) 2020 Magri, Stamatiou, Trinchieri and Perletti. This is an open-access article distributed under the terms of the Creative Commons Attribution License (CC BY). The use, distribution or reproduction in other forums is permitted, provided the original author(s) and the copyright owner(s) are credited and that the original publication in this journal is cited, in accordance with accepted academic practice. No use, distribution or reproduction is permitted which does not comply with these terms. 